\title{
Urine Dipstick Test
}

National Cancer Institute

\section{Source}

National Cancer Institute. Urine Dipstick Test. NCI Thesaurus. Code C147905.

Use of a test strip designed to detect one or more specific substances in urine. Results are indicated by a color change and may be qualitative or semi-quantitative. 